\title{
Modelamiento de la eficiencia productiva de trozas de lenga (Nothofagus pumilio) destinadas a la producción de madera aserrada en la Patagonia chilena
}

\author{
Modeling productive efficiency of lenga (Nothofagus pumilio) logs for \\ solid wood production in the Chilean Patagonia
}

Rosa M. Alzamora', Antonio Pinto2, Carolina Ulloa ${ }^{3}$ y Alfredo Aguilera ${ }^{4}$

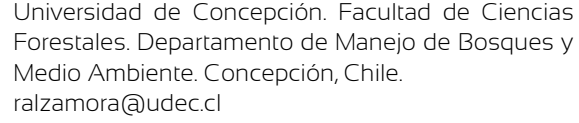
Forestales. Departamento de Manejo de Bosques y Medio Ambiente. Concepción, Chile. ralzamora@udec.cl

\author{
2 Universidad de Concepción. Facultad de Agronomía \\ Departamento de Producción Vegetal. Concepción, \\ Chile \\ Predio Monte Alto, XII Región \\ c.ulloa@sapatagonia.c
}

\section{RESUMEN}

Un análisis productivo y económico fue realizado sobre una muestra de 184 trozas de lenga (Nothofagus pumilio) obtenidas en el Predio Monte Alto, XII Región, Chile. Las trozas se destinaron a producir las calidades: selecta (S) y común (C1, C2 y C3), y maderas cortas de ancho variable. El análisis de eficiencia productiva se realizó utilizando dos fronteras de producción estocásticas (FE) tipo Cobb-Douglas y Translog. Este enfoque permite modelar funciones de producción y obtener medidas de eficiencia técnica $\left(\mathrm{EF}_{\mathrm{t}}\right)$. Los atributos de troza usados en los modelos fueron diámetro menor, largo y la productividad del proceso. $\mathrm{La}_{\mathrm{EF}}$ promedio de las trozas fue 0,85 y 0,78 calculada con FE Cobb-Douglas y Translog, respectivamente, lo que implica que en promedio las trozas podrían reducir el uso de los insumos en $15 \%$ o $22 \%$ y alcanzar el mismo nivel de producción. Las trozas con mayor $\mathrm{EF}_{\mathrm{t}}(\geq 0,9)$ se caracterizaron por presentar un diámetro menor promedio de $33 \mathrm{~cm}$ en ambos modelos y por generar un valor de recuperación promedio de USD $33 \mathrm{~m}^{-3}$ (a precios de 2017) y USD $29 \mathrm{~m}^{-3}$, respectivamente. Hubo correlaciones positivas y significativas entre la eficiencia, el volumen de los productos de mayor precio y el valor residual de las trozas $(r=0,53 ; p \leq 0,05)$.

PALABRAS CLAVE: aserrío, atributos, bosque nativo, frontera estocástica, valor residual de la troza

\section{ABSTRACT}

A productive and economic analysis was conducted on a sample of 184 logs of lenga (Nothofagus pumilio) from Monte Alto Land, XII Region, Chile. Logs were allocated to produce select (S), regular (C1, C2 and C3), and short lumber qualities. The productive efficiency analysis was done by production stochastic frontiers Cobb-Douglas and Translog. This approach is based on production functions and generates technical efficiency measures $\left(\mathrm{EF}_{\mathrm{t}}\right)$ to assess production systems. Logs attributes used as inputs were small end diameter, length and process productivity. The average $\mathrm{EF}_{\mathrm{t}}$ values of logs were 0,85 and 0,78 calculated by Cobb-Douglas and Translog frontiers, respectively. These measures imply that, on average, the logs could use reduced attributes by $15 \%$ or $22 \%$ and still achieve the same output. The most efficient logs $\left(\mathrm{EF}_{\mathrm{t}} \geq 0,9\right)$ were characterized by obtaining an average small end diameter of $33 \mathrm{~cm}$, and an average recovery value of USD $33 \mathrm{~m}^{-3}$ and $29 \mathrm{~m}^{-3}$, for Cobb-Douglas and Translog frontiers, respectively. There were significant positive correlations between efficiency and the logs recovery values $(\mathrm{r}=0,53 ; p \leq 0,05)$.

KEYWORDS: sawmill, attributes, native forest, stochastic frontier, residual log value 


\section{INTRODUCCIÓN}

Los bosques de lenga (Nothofagus pumilio [Poepp. Et Endl] Krasser) se extienden en un área de 3,6 millones de hectáreas, lo que representa $27 \%$ de la superficie con bosque nativo en Chile. Estos bosques se encuentran mayoritariamente en la región de Magallanes y Antártica con una superficie aproximada de 1,3 millones de hectáreas. Además de su extensión, la lenga es el recurso nativo comercial más importante de Chile; así, del total de exportaciones derivadas del bosque nativo, la lenga representa $63 \%$ con un valor de USD $\$ 3.5$ millones FOB (Instituto Forestal [Infor], 2017). Esto se debe a que el patrimonio concentrado en las regiones XI y XII es capaz de mantener una oferta sostenida de trozas para generar productos que mayoritariamente se van al mercado externo. Los valores de exportación para los principales productos de lenga durante el periodo 2009-2016 se concentran en madera aserrada, tablones y las partes y piezas de muebles tienen la mayor participación durante el periodo analizado con $43 \%$ y $38 \%$ respectivamente (Infor, 2017).

La madera de lenga se caracteriza por un color café claro a grisáceo con líneas amarillentas, rosadas y verdosas con una veta muy uniforme y es valorada por su versatilidad para distintos usos (Pérez, 1983; Schmidt y Caldentey, 1994).

El manejo y aprovechamiento de esta especie se realiza mediante cortas de protección; al respecto, Schmidt y Caldentey (1994) afirman que estas cortas son las más recomendables para la sustentabilidad del recurso. Ampuero (1995) y Duhalde (2002) indican que las cortas de protección permiten una mayor extracción y aprovechamiento del volumen aserrable y reducen los costos de producción. No obstante, existe una alta proporción de individuos que tiene pudrición central, lo que reduce su valor de recuperación; además, es difícil identificar el problema previo a la cosecha y procesamiento de los trozos, por ello, los estudios aluden a porcentajes de aprovechamiento del orden de $25 \%$ a $35 \%$ (Donoso y Caldentey, 1995). Por su parte, Schmidt y Urzúa (1982) estimaron que del bosque se extrae entre $10 \%$ a $20 \%$ de las existencias como volumen aserrable, y de este, solo $30 \%$ es convertido en madera aserrada. La pudrición blanca y café es la que predomina en los bosques de lenga (Pesutic, 1978). No obstante, en el presente, silvicultores chilenos y argentinos utilizan sistemas de clasificación de trozas que incluyen la presencia de signos de pudrición en las caras (Ulloa, 2013). En cuanto a la calidad de la troza, esta es altamente dependiente de su tamaño y rectitud dada la influencia de estas en la recuperación de madera aserrada. Respecto al largo de las trozas de lenga, Donoso y Caldentey (1996) señalan que este varía de dos metros a cuatro metros y que tiene una alta ponderación en la conversión a madera aserrada. Así, la conversión de volumen bruto a volumen aserrable estaría estrechamente relacionada con el tamaño y curvatura del trozo (Velasco, 1992). Donoso y Caldentey (1996) señalan que el rendimiento de las trozas de lenga está determinado por el diámetro menor, la sanidad y la rectitud.

En la producción maderera, los atributos de las trozas tienen el rol de insumos y están altamente relacionados con el volumen y la calidad de madera de los productos finales; así, tener unidades extra de atributos o menores defectos en las trozas generaría cambios cuantitativos y cualitativos en la producción y en el valor de recuperación de las mismas. $\mathrm{Al}$ respecto, Groen (2003) señala que los atributos de las materias primas se relacionan con la eficiencia económica de producción, por ello, es relevante cuantificarlos y valorarlos. Por otra parte, obtener información de atributos madereros de trozas (y árboles) es complejo debido a su natural heterogeneidad (Alzamora, Apiolaza y Evison, 2013). En el caso de lenga, aun considerando la pudrición, se requiere clasificar y segregar la materia prima en trozas para incrementar la eficiencia operativa del proceso y para maximizar su valor de recuperación. Un enfoque que resulta interesante al momento de evaluar la eficiencia productiva de las trozas es el de las fronteras de eficiencia (FE). Estas se basan en la modelación de funciones de producción para obtener medidas de eficiencia en las relaciones insumo-producto. Una de las FE es el análisis envolvente de datos (DEA, por sus siglas en inglés) que se 
basa en programación lineal y genera funciones de producción no paramétricas sobre los datos; así, las medidas de eficiencia son estimadas en relación con dicha frontera (Coelli, Rao, O'Donell y Battese, 2005). Todoroki y Carson (2003) aplicaron un enfoque DEA para identificar las trozas más eficientes para producir madera de apariencia con el fin de apoyar los patrones de selección en programas de mejoramiento genético. La otra FE es la estocástica que permite generar un modelo paramétrico de productos e insumos y obtener medidas de eficiencia técnica o productiva (Aigner, Lovell y Schmidt, 1977; Meeusen y van den Broeck, 1977; Coelli et al., 2005). Alzamora, Apiolaza y Evison (2013) estimaron fronteras estocásticas del tipo Cobb Douglas y Translog, para modelar la relación entre características de trozas y recuperación de madera estructural. Siguiendo esta línea, el presente estudio evalúa la factibilidad de identificar y caracterizar el perfil de atributos de las trozas de lenga a través de un enfoque de eficiencia productiva. Con esto, se espera aportar información en materia de procesamiento, rendimiento y retornos económicos, así como de la variabilidad productiva de esta especie.

\section{OBjetivos}

Para el presente estudio se planteó: 1) documentar los rendimientos y el valor de recuperación a partir de un estudio realizado en los bosques de lenga de Magallanes; 2) analizar las relaciones entre productos y atributos de trozas; y 3) aplicar un análisis de eficiencia productiva con la FE estocástica para evaluar la eficiencia productiva de las trozas.

\section{MATERIALES Y MÉTODOS}

Una muestra de 184 trozas lenga fue obtenida desde un rodal perteneciente a la Sociedad Monte Alto Forestal S.A., en la Provincia de Última Esperanza, región de Magallanes, Chile. La elevación del rodal era de $450 \mathrm{~m}$ snm y la exposición sur oeste. El rodal estaba en la etapa de envejecimiento con regeneración en estado de latizal alto y fustal delgado, por ello, existía una alta variabilidad en las edades, estimándose un mínimo de 20 años y un máximo de 80 años. Los árboles fueron seleccionados con base en rectitud y altura, procurando aprovechar una troza aserrable de siete pies $(2,13 \mathrm{~m})$. El intervalo de diámetros a la altura de pecho (DAP) era de $25 \mathrm{~cm}-65 \mathrm{~cm}$. Se seleccionaron, marcaron y rotularon diez árboles por clase de DAP en intervalos de $5 \mathrm{~cm}$. La rotulación consideró DAP, altura total y altura comercial que, para las especies delicuescentes como lenga, equivale a la altura donde comienzo la copa. El trozado se realizó en cancha de acopio, a $15 \mathrm{~km}$ del rodal. Aquí, cada troza fue medida e identificada con el árbol al cual pertenecía (Tabla 1). Las trozas se cubicaron con la fórmula de Smalian [1].

TABLA 1. Volumen promedio $(\mathrm{Vm})$ y $L$ promedio de ingreso de trozas por clase diamétrica.

\begin{tabular}{cc|cc|cc}
\hline $\begin{array}{c}\text { Clase diámetro } \\
\text { menor }(\mathrm{cm})\end{array}$ & $\begin{array}{c}\text { Muestras } \\
\left(\mathrm{N}^{0}\right)\end{array}$ & $V m\left(\mathrm{~m}^{3} /\right.$ troza) & $\begin{array}{c}\text { Desv. } \\
\text { Estándar }\left(\mathrm{m}^{3}\right)\end{array}$ & $\begin{array}{c}\text { L promedio } \\
(\mathrm{m} / \text { troza })\end{array}$ & $\begin{array}{c}\text { Desv. } \\
\text { Estándar }\left(\mathrm{m}^{3}\right)\end{array}$ \\
\hline $18-21$ & 13 & 0,12 & 0,03 & 3,34 & 0,51 \\
$21,1-24$ & 37 & 0,18 & 0,05 & 3,47 & 0,57 \\
$24,1-27$ & 24 & 0,21 & 0,05 & 3,30 & 0,51 \\
$27,1-30$ & 36 & 0,29 & 0,07 & 3,43 & 0,63 \\
$30,1-33$ & 15 & 0,32 & 0,09 & 3,38 & 0,60 \\
$33,1-36$ & 18 & 0,40 & 0,08 & 3,42 & 0,60 \\
$36,1-39$ & 8 & 0,57 & 0,14 & 3,97 & 0,68 \\
$39,1-42$ & 15 & 0,47 & 0,11 & 3,13 & 0,55 \\
$42,1-45$ & 7 & 0,68 & 0,20 & 3,60 & 0,95 \\
$45,1-48$ & 7 & 0,67 & 0,19 & 3,23 & 0,75 \\
$>48$ & 4 & 0,88 & 0,25 & 3,52 & 0,91 \\
\hline
\end{tabular}




$$
V=\left\{\frac{(A+a)}{2}\right\} * L
$$

donde:

$V$ es el volumen de la sección en $\mathrm{m}^{3}$

$A$ es el área transversal de la sección de mayor diámetro $\left(\mathrm{m}^{2}\right)$

$A=\pi \frac{d^{2}}{4}, a$ es el área transversal de diámetro menor $\left(\mathrm{m}^{2}\right)$

L es el largo de la sección (m)

Además, se registró la productividad desde que una troza caía al carro transportador hasta que la próxima troza ingresaba al mismo.

El aserradero era fijo, semiautomático y trabajaba a un turno, componiéndose de las siguientes unidades: carro transportador (uno), reaserradora (uno), canteadora (uno) y despuntadora (uno). El aserradero no operaba con programas específicos de corte, sino que el operador iba aplicando su criterio de aprovechamiento para maximizar el ancho de la pieza en la canteadora. Así, la madera de lenga producida era de ancho variable permitiendo recuperar el máximo del volumen de la troza. Esta es una estrategia de producción para manejar el problema de las pudriciones que caracteriza a esta especie.

Una vez clasificadas las tablas se les registró su espesor (pulgadas), ancho (pulgadas) y largo (pies). La clasificación de calidad se basó en la norma National Hardwood Lumber Association (NHLA). Luego se determinó el valor de recuperación de las trozas (VR, USD $\mathrm{m}^{-3}$ ), que se calcula como la diferencia entre el ingreso por la venta de la madera producida a partir de $1 \mathrm{~m}^{3}$ de troza y los costos de procesamiento (Davis, Johnson, Bettinger y Howard, 2001). Para obtener VR se utilizaron los precios detallados en la tabla 2, y el costo total de operación fue facilitado por la empresa Sociedad Monte Alto y era de USD $49 \mathrm{~m}^{-3}$ (Ulloa, 2013). La calidad selecta (S) se caracteriza por piezas largas libres de defectos y es principalmente usada para molduras decorativas y cubiertas de mesa. Estas piezas se clasifican por su rendimiento de superficie limpia por la mejor cara $(83 \%-100 \%)$ y peor cara $(67 \%-83 \%)$.
TABLA 2. Precios de venta de madera aserrada.

\begin{tabular}{lll}
\hline Calidad & $\begin{array}{l}\text { Precio* } \\
\left(U S D \mathrm{~m}^{-3}\right)\end{array}$ & Destino \\
\hline Calidad Selecta (S) & 309,36 & Exportación \\
Primera Común (C1) & 200,07 & Nacional \\
Segunda Común (C2) & 116,55 & Nacional \\
Tercera Común (C3) & 64,38 & Regional \\
Media (Md) & 129,09 & Regional \\
Media inferior (Md1) & 58,11 & Regional \\
Maderas Cortas (C) & 70,98 & Regional \\
Construcción (O-P) & 261,37 & Regional \\
\hline $\begin{array}{l}\text { * Dólares americanos a precios del año 2017, no incluyen IVA ni costo de } \\
\text { transporte. }\end{array}$ &
\end{tabular}

El análisis de eficiencia productiva de las trozas se realizó con fronteras estocásticas utilizando los modelos CobbDouglas y Transcendental Logarítmica (Translog). La estimación de estas fronteras considera dos errores, el aleatorio y el de ineficiencia, para el último se asume una distribución normal truncada (Coelli, 2005). La función de producción Cobb-Douglas se define por la siguiente relación [2]:

$$
Q=\beta_{0} \Pi_{k=1}^{m} X_{k}^{\beta_{k}} \quad k=1, \ldots, \mathrm{m}
$$

donde $Q$ es el producto total y $X_{k}$ son los factores de producción. En este tipo de modelo, el coeficiente $\beta_{k}$ corresponde a la elasticidad producto del factor e indica el porcentaje de cambio en el producto total cuando el insumo k varía en 1\% (Varian, 2003).

La función Translog representa un modelo de producción más flexible que permite evaluar elasticidad de sustitución variable entre insumos. Su forma funcional es [3]:

$$
\ln Q=\beta_{0}+\sum_{k=1}^{m} \beta_{k} \ln X_{k}+1 / 2 \sum_{k=1}^{m} \sum_{l=1}^{m} \beta_{k l} \ln X_{k} \ln X_{l}
$$

donde $Q$ es el producto total y $X_{k}$ son los insumos y los $\beta_{k}$ corresponden a los coeficientes del modelo. 
Para fines de este estudio, el producto correspondió al volumen por metros cúbicos de troza producido en todas las calidades que se detallan en la tabla 2. En cuanto a los insumos, se utilizaron los atributos: largo $(L, \mathrm{~m})$ y diámetro menor de las trozas $(D m, \mathrm{~cm})$. La variable $L$ se incluyó porque fue un atributo variable en el procesamiento de las trozas y es determinante para la estimación de volumen, al igual que $D m$, la cual ha demostrado ser una variable muy significativa en la recuperación de volumen de productos. Aunque la materia prima de lenga se caracteriza por problemas de pudrición central estos no fueron registrados en el estudio. Por ello, se utilizó la productividad del proceso $\left(\mathrm{m}^{3} / \mathrm{h}\right)$ como una aproximación inversa al efecto que se observaría por las pudriciones. Así, en la medida en que hay más problemas de pudrición, el operario de la canteadora demorará más en ajustar la troza al proceso para maximizar el ancho de las piezas.

\section{Resultados}

La madera de calidad primera común (C1) presentó la mayor participación a través de todas las clases diamétricas con un promedio de 31,7\% (Tabla 3). En tanto que la madera común C2 presentó una participación promedio de $19 \%$, y no se observó una tendencia definida en su distribución por clase diamétrica. En general, la calidad media (Md) y la de escuadrías cortas (Cr) se produjeron en bajos porcentajes $7,7 \%$ y $2,4 \%$, sin una relación definida con el $D m$ de las trozas. Estas escuadrías se generaron con el objetivo de aprovechar al máximo el trozo con largos mínimos de 0,9 m-1,5 m, respectivamente. Las maderas de construcción ( $\mathrm{O}$ y $\mathrm{P}$ ) mostraron una participación muy baja $(\leq 2 \%)$, lo que se explicaría debido a que estas son de ancho fijo y la estrategia general de proceso fue ancho variable.

La tabla 4 presenta un resumen del rendimiento aserrable de las trozas y de la participación de los productos. El volumen de los productos de mayor valor, $\mathrm{S}$ y $\mathrm{C} 1$, estuvo positivamente correlacionado con el diámetro menor de la troza, $\mathrm{r}=0,58 \mathrm{y} \mathrm{r}=0,66(p \leq 0,001)$ respectivamente; no obstante, el producto C3 se correlacionó en forma negativa con el $\mathrm{Dm}$.

TABLA 3. Volumen promedio y rendimiento de trozas en los distintos productos.

\begin{tabular}{|c|c|c|c|c|c|c|c|c|c|c|}
\hline \multirow{2}{*}{$\begin{array}{l}\text { Diámetro } \\
\text { menor }(\mathrm{cm})\end{array}$} & \multirow{2}{*}{$\begin{array}{l}N^{\circ} \text { de } \\
\text { muestras }\end{array}$} & \multirow{2}{*}{$\begin{array}{c}\text { Volumen } \\
\text { troza } \\
\left(m^{3} / \text { troza }\right)\end{array}$} & \multirow{2}{*}{$\begin{array}{l}\text { Rendimiento } \\
\text { (\%) }\end{array}$} & \multicolumn{7}{|c|}{ Porcentaje de participación por calidad* } \\
\hline & & & & $s$ & $\mathrm{Cl}$ & $C 2$ & C3 & $O-P$ & $M d$ & $\mathrm{Cr}$ \\
\hline $18-21$ & 13 & 0,12 & 36,4 & 0,0 & 12,5 & 26,0 & 44,2 & 0,0 & 11,2 & 6,1 \\
\hline $21,1-24$ & 37 & 0,18 & 39,3 & 10,1 & 22,5 & 19,8 & 31,4 & 4,2 & 8,9 & 3,1 \\
\hline $24,1-27$ & 24 & 0,21 & 43,0 & 11,7 & 28,2 & 14,6 & 29,1 & 0,0 & 12,3 & 4,2 \\
\hline $27,1-30$ & 36 & 0,29 & 42,8 & 11,2 & 34,5 & 17,7 & 20,7 & 3,1 & 9,6 & 3,3 \\
\hline $30,1-33$ & 15 & 0,32 & 46,8 & 22,4 & 36,5 & 15,0 & 11,6 & 0,0 & 10,7 & 3,8 \\
\hline $33,1-36$ & 18 & 0,40 & 43,3 & 15,1 & 38,7 & 14,4 & 21,5 & 1,7 & 7,1 & 1,6 \\
\hline 36,1 - 39 & 8 & 0,57 & 45,9 & 28,3 & 37,9 & 12,2 & 16,4 & 2,0 & 3,2 & 0,0 \\
\hline $39,1-42$ & 15 & 0,47 & 49,7 & 27,4 & 30,2 & 24,0 & 10,4 & 2,1 & 5,2 & 0,7 \\
\hline $42,1-45$ & 7 & 0,68 & 46,4 & 18,0 & 50,8 & 13,0 & 9,4 & 2,1 & 5,2 & 1,5 \\
\hline $45,1-48$ & 7 & 0,67 & 50,4 & 23,8 & 28,0 & 31,8 & 10,8 & 1,1 & 3,0 & 1,6 \\
\hline$>48$ & 4 & 0,88 & 32,4 & 36,1 & 29,4 & 19,4 & 6,5 & 0,0 & 8,6 & 0,0 \\
\hline Total & 184 & 0,33 & 43,0 & 18,5 & 31,7 & 18,9 & 19,3 & 1,5 & 7,7 & 2,4 \\
\hline
\end{tabular}

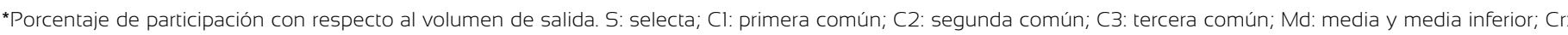
cortas; O-P: maderas de construcción. 
TABLA 4. Correlaciones entre VR, atributos de las trozas, variables de proceso y productos.

\begin{tabular}{|c|c|c|c|c|c|}
\hline & $\begin{array}{c}V R \\
\left(U S D m^{-3}\right)\end{array}$ & $\begin{array}{l}\text { Dm troza } \\
\quad(\mathrm{cm})\end{array}$ & $\begin{array}{c}\text { Rendimiento } \\
(\%)\end{array}$ & $\begin{array}{l}\text { L troza } \\
(m)\end{array}$ & $\begin{array}{c}\text { Productividad } \\
\qquad\left(m^{3} / h\right)\end{array}$ \\
\hline$D m$ troza $(\mathrm{cm})$ & $0,47^{* * *}$ & & & & \\
\hline Rendimiento (\%) & $0,76^{* * *}$ & $0,18^{* *}$ & & & \\
\hline$L$ troza $(\mathrm{m})$ & $-0,09$ ns & 0,05 ns & $-0,15^{*}$ & & \\
\hline Productividad $\left(\mathrm{m}^{3} / \mathrm{h}\right)$ & $0,65^{* * *}$ & $0,45^{* * *}$ & $0,63^{* * *}$ & 0,07 ns & \\
\hline$S\left(m^{3}\right)$ & $0,62^{* * *}$ & $0,58^{* * *}$ & $0,21^{* *}$ & 0,03 ns & $0,33^{* * *}$ \\
\hline $\mathrm{Cl}\left(\mathrm{m}^{3}\right)$ & $0,59^{* * *}$ & $0,66^{* * *}$ & $0,36 * * *$ & $0,26^{* *}$ & $0,51^{* * *}$ \\
\hline $\mathrm{C} 2\left(\mathrm{~m}^{3}\right)$ & $0,14^{*}$ & $0,47^{* * *}$ & $0,22^{* *}$ & $0,16^{* *}$ & $0,17^{* *}$ \\
\hline $\mathrm{C} 3\left(\mathrm{~m}^{3}\right)$ & $-0,19^{* *}$ & $-0,01 \mathrm{~ns}$ & $0,22 * *$ & $0,18^{* *}$ & $0,23^{* *}$ \\
\hline
\end{tabular}

*** nivel de significancia $p \leq 0,001$, ** nivel de significancia $p \leq 0,05, y$ * nivel de significancia $p \leq 0,1$

\section{Relación ente el valor de recuperación de las trozas, sus atributos y procesamiento}

El VR promedio de las todas trozas fue de USD $19 \mathrm{~m}^{-3}$, el mayor valor fue USD $104 \mathrm{~m}^{-3}$. Estos valores son comparables a los señalados para otras especies más comerciales como Pinus radiata D. Don. Alzamora y Apiolaza (2010) observaron valores de hasta USD $114 \mathrm{~m}^{-3}$ para trozas podadas de $P$. radiata destinadas a la producción de grados de apariencia.

Acorde con lo esperado y según lo indica la tabla 4, el $D m$ de la troza y el rendimiento presentaron una relación directa con el VR; así mismo, al incrementarse el rendimiento de producción, todo el volumen comercial de productos aumenta; esto explicaría la correlación positiva y significativa, $0,76(p \leq 0,001)$, así como la correlación con $\mathrm{S}$ y $\mathrm{C} 1$.

\section{Análisis de eficiencia}

Los resultados de la frontera de producción estocástica CobbDouglas con error half-normal se presentan en la tabla 5 .

El modelo muestra que los parámetros asociados a $D m, L$ y productividad tuvieron un efecto positivo y significativo sobre el producto total. En ese sentido, se observó que aumentos porcentuales de $D m$ incrementaban la producción en $1,65 \%$, mientras que $L$ y la productividad la aumentaron en $0,6 \%$ aproximadamente. Además, aumentos en $D m$ tuvieron un efecto que supera en 2,5 veces al $L$ de las trozas, por lo que deberían seleccionarse trozas de mayor diámetro si se desea mejorar la producción. Por otra parte, la relación con la productividad fue positiva, es decir, la disminución en el tiempo de procesamiento aumentó el rendimiento, sin embargo, esta es una relación indirecta puesto que dicho tiempo depende también de la condición de la troza o de los niveles de pudrición dada la estrategia de aserrío anteriormente descrita. Por ello, trozas con mejor perfil de calidad requerirán menos tiempo para ser procesadas y, a su vez, tendrán una alta correlación con productos tipo $\mathrm{S}$ y $\mathrm{C} 1$ que poseen mayor valor (correlación 0,33 y 0,51 respectivamente; Tabla 4).

La tabla 6 presenta los resultados del ajuste de la FE Translog, donde las pruebas de Sigma ${ }^{2}$ y Gamma permitieron inferir que el ajuste del modelo fue altamente significativo, $p \leq 0,001$. No obstante, la significancia de los parámetros varió entre $p \leq 0,1$ y $p \leq 0,001$; en ese sentido, el comportamiento de algunos parámetros, y en particular de aquellos que medían los efectos interactivos, fueron poco significativos; incluso, solo el parámetro que considera la interacción entre $D m$ y $L$ fue significativo $(p \leq$ $0,05)$. Esto quiere decir que el efecto positivo entre ambas variables, $D m$ y $L$, condiciona el efecto de cada una de las 
variables en particular, por ende, un aumento porcentual en $D m$ y su efecto en la producción frontera dependerá del nivel en el cual esté el $L$ de la troza; así, en la medida en que mayor sea el $L$ de las trozas, el efecto de aumento en $D m$ se verá marginalmente incrementado, por lo que habría un efecto positivo e incremental sobre la frontera de producción. Similar análisis se puede hacer ante aumentos del $L$ con $D m$. Ahora, el efecto individual tanto de $L$ como $D m$ sobre el nivel de aumento de la frontera de producción también dependerá del nivel o valor que cada una de las variables posea, y el efecto será incremental en la medida en que mayor sea el valor de cada una de estas. Finalmente, el efecto de la productividad sobre posibles desplazamientos de la frontera de producción, y en particular sobre sus posibles interacciones con otras variables, es complejo, ya que a medida en que la productividad aumenta o mejora la condición de la troza, hay efectos opuestos sobre la expansión de la frontera, y lo mismo se observa en la interacción de esta con $L$ o $D m$, que hacen que la frontera se expanda marginalmente menos, mientras mayores sean los valores observados en dichas variables. Si bien se observaría un efecto incremental sobre la frontera de producción, esta sería marginalmente menor mientras mayor (mejor) sea la condición de la troza (productividad), su diámetro menor o su longitud.

TABLA 5. Estimación de la función frontera de producción Cobb-Douglas.

\begin{tabular}{lcccc}
\hline & Coeficiente & Error estándar & t-valor & $p$-valor \\
\hline Intercepto & $-9,4570$ & 0,2722 & $-34,7460$ & $p \leq 0,001$ \\
$D m(\mathrm{~cm})$ & 1,6598 & 0,0749 & 22,1611 & $p \leq 0,001$ \\
L $(\mathrm{m})$ & 0,6409 & 0,0992 & 6,4576 & $p \leq 0,001$ \\
Productividad $\left(\mathrm{m}^{3} / \mathrm{h}\right)$ & 0,6017 & 0,0211 & 28,4731 & $p \leq 0,001$ \\
\hline Sigma & 0,0847 & 0,0190 & 4,4644 & $p \leq 0,001$ \\
Gamma & 0,4961 & 0,2004 & 2,4757 & $p \leq 0,05$ \\
\hline
\end{tabular}

Función Log de máxima verosimilitud =1,3447

Modelo fue ajustado con variables transformadas vía logaritmo natural

TABLA 6. Estimación de la función FE Translog.

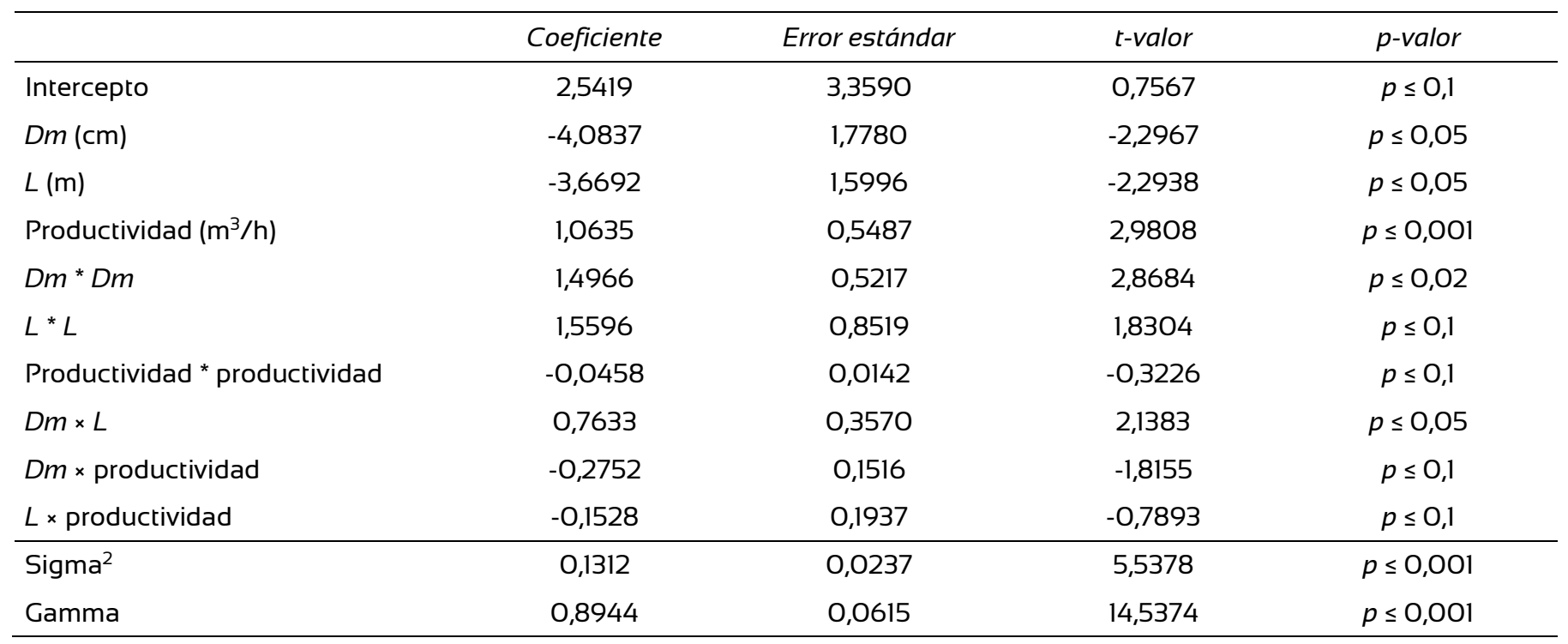

Función Log de máxima verosimilitud =11,8652

LR test de error de una cola $=9,1473$ 
A partir de FE Translog, 24 trozas presentaron niveles de $\mathrm{EF}_{\mathrm{t}} \geq 0,9$, y el $D m$ promedio de estas trozas $33,2 \mathrm{~cm}$, concentrando $58 \%$ de estas en $D m \geq 30 \mathrm{~cm}$; además, en los menores $D m$, la troza que presentó mayor eficiencia fue de $20 \mathrm{~cm}$. Por otra parte, el VR de las trozas más eficientes fue de USD $33 \mathrm{~m}^{-3}$. En cuanto a la relación con $L$, las trozas más eficientes presentaron un promedio de $3,55 \mathrm{~m}$.

Respecto a los niveles de eficiencia que se obtuvieron con la FE Cobb-Douglas, 27 trozas presentaron niveles de $\mathrm{EF}_{\mathrm{t}} \geq 0$,9. El $D m$ promedio de dichas trozas fue $33,5 \mathrm{~cm}$, concentrando $52 \%$ de estas en $D m \geq 30 \mathrm{~cm}$, el VR de las trozas más eficientes fue de USD 28,9 $\mathrm{m}^{-3}$. En cuanto a la relación con $L$, las trozas más eficientes presentaron un promedio de $3,45 \mathrm{~m}$.

Por otra parte, los niveles de eficiencia alcanzados variaron de acuerdo con las diferentes clases de $L$ y $D m$. Respecto a $D m$ no se observó una tendencia clara sobre cambios en la variabilidad de la eficiencia en la medida que se incrementa la clase de diámetro; no obstante, con $L$ sí se observó que en las clases superiores la variabilidad de la eficiencia tendió a disminuir y la eficiencia se concentró en valores superiores a 0,8 .

\section{DISCUSIÓN}

Lenga es actualmente la madera nativa chilena más comercializada y más conocida y apreciada en el extranjero. En 2016 sus productos generaron USD \$3,1 millones FOB, lo que implica $91,3 \%$ de participación entre las exportaciones derivadas de bosques naturales; aunque según Infor (2017), en ese mismo año, las exportaciones de lenga bajaron 20,7\% respecto al año 2015. Los productos de lenga con mayor participación ( $80 \%$ del monto FOB) fueron madera aserrada y madera cepillada con USD 1,3 $\times$ $10^{6}$ y USD 1,2 $\times 10^{6}$, respectivamente. En relación con los destinos, los tres primeros lugares de la tabla de clasificación mundial son Ecuador (30\% del monto de 2016), China (20\%) y Estados Unidos (14\%). El hecho de que los productos de lenga sean reconocidos y valorados, impulsa mejoras para desarrollar modelos de manejo silvícola sustentables y concentrados en recuperar valor del bosque. Es así que, se reconoce que en una perspectiva de largo plazo el mejor método de manejo es la corta de protección. Caldentey (1994); Ampuero (1995); Duhalde (2002); Peri, Martínez y Monelos (2013) y Otero y Vergara (2016) afirman que este sistema promueve una mayor sustentabilidad, con mejores posibilidades de extracción y aprovechamiento del volumen aserrable.

Tanto para lenga como para otras especies del bosque nativo, las magnitudes de producción son generalmente analizadas por unidad de superficie (hectárea); sin embargo, el presente estudio enfocó la unidad de producción a las trozas, por la íntima relación que existe entre los atributos de esta y la calidad y el volumen de productos que se pueden generar. Por ello, el interés del trabajo se centró en analizar el potencial productivo de las trozas a través de las fronteras de eficiencia para caracterizar el perfil de atributos que genera una producción eficiente. Del mismo modo, en una muestra de 184 trozas se determinó su valor de recuperación (VR) para identificar su intervalo de variación ante distintos perfiles de atributos. El VR promedio de las trozas fue de USD $19 \mathrm{~m}^{-3}$, con un valor máximo de USD $104 \mathrm{~m}^{-3}$. Estos valores son comparables con los hallados para otras especies como Pinus radiata D.Don. De esta forma, Alzamora y Apiolaza (2010) obtuvieron valores de hasta USD $114 \mathrm{~m}^{-3}$ para trozas podadas de $P$. radiata destinadas a la producción de grados de apariencia.

De acuerdo con lo esperado, el diámetro menor de la troza y el rendimiento en aserradero presentaron una relación directa con el VR. Ulloa (2013), señala que a medida en que este diámetro aumenta, se incrementa la participación relativa en las calidades con mayor precio (selecta y primera común); así mismo, al incrementarse el rendimiento de producción, todo el volumen comercial de productos aumenta, lo cual se demuestra en la correlación positiva y significativa entre estas dos variables $(0,76 ; \mathrm{p} \leq$ 0,001).

El análisis de fronteras de eficiencia es raramente aplicado a las materias primas como las trozas; sin embargo, el estudio de Todoroki y Carson (2003) en trozas de $P$. radiata documentó significativas contribuciones para definir prioridades de mejora en las características de volumen y 
forma para efecto de producir grados de apariencia. En la misma línea, pero usando fronteras paramétricas, Alzamora et al. (2013) trabajaron en trozas de P. radiata para producir grados estructurales obteniendo niveles de eficiencia técnica comparables con los de lenga. Puede que este resultado sorprenda, sin embargo, en el estudio de P. radiata se registraron valores muy bajos de módulo de elasticidad dinámico, atributo que es fundamental para definir la calidad estructural. En los resultados de este estudio, las trozas con mayor volumen relativo de productos selecta y primera común, fueron las que obtuvieron el máximo puntaje de eficiencia. Sin embargo, no hubo trozas con $\mathrm{EF}_{\mathrm{t}}$ $=1$ en ambos modelos ajustados, lo cual podría ser explicado por la heterogeneidad de las trozas, la cual tendió a aumentar el error estocástico. La frontera estocástica Translog, resultó en 24 trozas con niveles de $\mathrm{EF}_{\mathrm{t}} \geq 0,9$ y $\mathrm{Dm} \geq 30 \mathrm{~cm}$; así mismo, el diámetro menor de las trozas que generó la mayor eficiencia fue de $20 \mathrm{~cm}$, el cual está dentro de los límites obtenidos por Ulloa (2013) quien con base en estadígrafos paramétricos y valores de recuperación determinó que el diámetro menor mínimo debía ser de $22 \mathrm{~cm}$.

Este análisis ofrece la posibilidad de ir hacia una mejora de corto plazo, porque entrega pautas para la caracterización de trozas con fines de sustentar una tecnología de segregación de trozas, lo cual debería ser el paso siguiente de esta línea de investigación. De igual modo, se requiere incorporar variables de sanidad, las cuales son relevantes en el caso de lenga, con el fin de ajustar modelos de producción que permitan predecir volúmenes y calidades, lo cual será parte de futuras investigaciones.

\section{CONCLUSIONES}

Existe una relación positiva entre el aumento en la recuperación del producto y el volumen de la troza; similar relación se observa con el volumen de mayor calidad y valor (selecta).

En promedio, se observó 18,5\% de calidad selecta, $31,7 \%$ calidad primera común, $18,9 \%$ calidad segunda común y 19,3\% calidad tercera común.

Las trozas con mayores índices de eficiencia $\left(\mathrm{EF}_{\mathrm{t}} \geq\right.$ 0,9) generados con la Translog y la Cobb-Douglas, fueron las que generaron el mayor volumen de calidad selecta y primera común, las que a su vez son los productos de mayor valor.

La modelación de la producción de madera de lenga a través del enfoque de fronteras de producción es un medio eficiente para plantear tecnologías de segregación de trozas en aserradero y para focalizar los atributos de valor en el manejo del bosque.

Futuras investigaciones deberían estudiar el efecto específico de los diferentes niveles de pudrición en las trozas de lenga, los productos obtenidos y, consecuente a ello, proponer un índice para segregar.

\section{REFERENCIAS}

Aigner, D., Lovell, C., \& Schmidt, P. (1977). Formulation and estimation of stochastic frontier production function models. Journal of Econometrics, 6(1), 21-37. doi: 10.1016/0304-4076(77)90052-5

Alzamora, R., Apiolaza, L., \& Evison, D. (2013). Using a production approach to estimate economic weights for structural attributes of Pinus radiata wood. Scandinavian Journal of Forest Research, 28(3), 282-290. doi: 10.1080/02827581.2012.734328

Alzamora, R., \& Apiolaza, L. (2010). A hedonic approach to value Pinus radiata $\log$ traits for appearance-grade lumber production. Forest Science, 53(6):283-291. doi: 10.1093 / forestscience/56.3.281

Ampuero, T. (1995). Rendimiento de la lenga (Nothofagus pumilio Poepp. Endl. Krasser) en el aserradero y su relación con las características de los árboles (Tesis de pregrado). Universidad de Chile, Santiago, Chile.

Coelli, T., Rao D., O' Donell, C., \& Battese, G. (2005). An introduction to efficiency and productivity analyses. New York: Springer.

Davis, L., Johnson, K., Bettinger, P., \& Howard, T. (2001). Forest Management (4a ed.). New York: McGraw-Hill.

Donoso, C. \& Caldentey, J. (1996). Rendimiento de la lenga (Nothofagus pumilio Poepp. Endl. Krasser) en el aserradero y su relación con las características de los árboles. Forestales, 11(1-2), 19-30.

Donoso, C., \& Caldentey, J. (1995). Rendimiento de lenga (Nothofagus pumilio) en el aserrado y su relación con las características silvicolas de los árboles. IV Jornadas Forestales Patagónicas. San Martín de los Andes, Argentina. Recuperado de 
http://revistacienciasforestales.uchile.cl/1996_vol11/n12a2.pdf

Duhalde, P. (2002). Análisis físico de la intervención de corta final a nivel experimental en dos rodales de lenga (Nothofagus pumilio (Poepp. et Enda.) Krasser) en la zona de Magallanes: un caso de estudio (Tesis pregrado). Universidad Austral, Valdivia, Chile.

Groen, A. (2003). Poultry genetics, breeding and biotechnology. Trowbridge, UK: Cromwell Press.

Instituto Forestal [INFOR]. 2017. Anuario Forestal 2017. Boletín Estadístico $\mathrm{N}^{\circ} 159$. Chile: INFOR. Recuperado de http://wef.infor.cl/publicaciones/anuario/2017/Anuario2 017.pdf

Meeusen, W. \& Van Den Broeck, J. (1977). Efficiency estimation for Cobb-Douglas production functions with composed error. International Economic Review, 18, 435-444.

Otero, L. \& Vergara, G. (2016). Efectos de diferentes métodos de corta de bosque nativo en el paisaje. Sustainability, Agri, Food and Environmental Research, 4(2). doi: 10.7770/saferV4N2-art993

Peri, P., Martínez, G., \& Monelos, L. (2013). Natural dynamics and thinning response of young lenga (Nothofagus pumilio) trees in secondary forests of Southern Patagonia. Bosque, 34(3), 273-279. DOI: 10.4067/S0717-92002013000300003

Pérez, V. (1983). Manual de propiedades físicas y mecánicas de maderas chilenas. Documento de Trabajo $\mathrm{N}^{\mathrm{o}} 47$, Proyecto CONAF/PNUD/FAO-CHI 76/003. Santiago, Chile.

Pesutic, S. (1978). Análisis de estructura-estado sanitario en un bosque de lenga. Ingeniero forestal (Tesis pregrado). Universidad de Chile. Santiago, Chile.

Schmidt, H., \& Urzúa, A. (1982). Transformación y manejo de los bosques de lenga en Magallanes. Santiago, Chile: Universidad de Chile.
Schmidt, H. \& Caldentey, J. (1994). Apuntes tercer curso silvicultura de los bosques de lenga. Universidad de Chile, CORMA Austral y Corporación Nacional Forestal.

Todoroki, C. \& Carson, S. (2003). Managing the future forest resource through designer trees. International Transactions in Operational Research, 10(5), 449-460. doi: 10.1111/14753995.00421

Ulloa, C. (2013). Obtención del diámetro minimo para trozas de lenga (Nothofagus pumilio) destinada a la producción de madera aserrada en el predio Monte Alto, XII Región (Tesis pregrado). Universidad Austral, Valdivia, Chile.

Van Biesebroeck, J. (2007). Robustness of productivity estimates. The Journal of Industrial Economics, 55(3): 529-569. doi: 10.1111/j.1467-6451.2007.00322.x

Varian, H. (2003). Intermediate microeconomics: a modern approach (6 ed.). New York: WW Norton.

Velasco, J. (1992). Rendimiento de madera aserrada libre de nudos en trozas podadas de Pinus radiata D. Don (Tesis pregrado). Universidad Austral, Valdivia, Chile.

Manuscrito recibido el 08 de marzo de 2018

Aceptado el 11 de abril de 2019

Publicado el 13 de diciembre de 2019

Este documento se debe citar como:

Alzamora, R. M., Ulloa, C., Pinto, A, \& Aguilera. A. (2019). Modelamiento de la eficiencia productiva de trozas de lenga (Nothofagus pumilio) destinadas a la producción de madera aserrada en la Patagonia chilena. Madera y Bosques, 25(3), e2531746. doi: $10.21829 /$ myb.2019.2531746

Madera y Bosques por Instituto de Ecología, A.C. se distribuye bajo una Licencia Creative Commons Atribución-NoComercialCompartirlgual 4.0 Internacional. 